\title{
Effective ultrasonic virtual sources which can be positioned independently of physical aperture focus positions
}

This article was published in the following Dove Press journal:

Reports in Medical Imaging

9 September 2010

Number of times this article has been viewed

\section{Chikayoshi Sumi \\ Shinya Uga}

Department of Information and Communication Sciences, Faculty of Science and Technology, Sophia University, Tokyo, Japan
Correspondence: Chikayoshi Sumi Department of Information and Communication Sciences, Faculty of Science and Technology, Sophia University, 7-I, Kioi Cho, Chiyoda Ku, Tokyo 102-8554, Japan

Tel +8I 3323834 I5

Fax +8I 33238332 I

Email c-sumi@sophia.ac.jp
Abstract: In this report, newly obtained ultrasonic virtual sources, VS1 and VS2, are described. That is, both virtual sources are obtained on the basis of the new viewpoint that the position of a virtual source can also be obtained regardless of the focus position of any physical aperture. VS1 is a virtual source that is a point acoustic source model set behind physical array elements and enables an increase in the transmitted ultrasound intensity using plural transmission ultrasound elements, and increases the size of the region of interest, that is, the same virtual source as that reported solo by other groups previously. As is well known, VS1 increases the echo signal-to-noise ratio; this yields a tissue displacement/strain measurement as well as B-mode imaging, and enables associated applications, eg, shear modulus reconstruction. Alternatively, VS2 uses an arbitrary position ahead of the physical array elements as a virtual source position with a synthetic aperture method. With VS2, random scattering media (material) such as point acoustic sources can be used as virtual sources. Preliminary experimental results are presented for a lateral modulation method. In the experiments described here using an ultrasound linear array type transducer (ultrasound element pitch, $0.2 \mathrm{~mm}$; ultrasound frequency, $7.5 \mathrm{MHz}$ ), it was possible to use a depth range of $\pm 3 \mathrm{~mm}$ for virtual source positions. Remarkably, VS2 yielded higher lateral resolutions than the physical sources. That is, VS2 mitigates the physical and electrical limitations for using smaller ultrasound array elements and a smaller pitch, such that both a high lateral resolution and a high echo signal-to-noise ratio are obtained.

Keywords: ultrasound, virtual source, lateral modulation, synthetic aperture, lateral resolution, displacement vector, strain tensor, shear modulus

\section{Introduction}

Effective ultrasound beamforming ${ }^{1-3}$ has been described using steered plural beams and apodizations for B-mode imaging with a high lateral resolution and for permitting accurate measurements of tissue (eg, heart and liver), blood displacement vectors, and/or strain tensors. ${ }^{3}$ This approach uses the multidimensional cross-spectrum phase gradient method, ${ }^{4}$ or multidimensional autocorrelation or Doppler method with multidimensional analytic signals. ${ }^{3}$

For such applications, in addition to the multidirectional synthetic aperture method $(\mathrm{MDSAM})^{3,5}$ and the multiple transmitting method, ${ }^{3,5}$ several lateral cosine modulation methods ${ }^{1-3}$ have been described, which yield the lateral frequency as well as the axial frequency by using different apodization functions, ie, functions expressed using separate Gaussian functions, Hanning windows and parabolic functions, and functions obtained with linear ${ }^{1,6}$ and/or nonlinear ${ }^{6}$ optimization so that a designed, appropriate point spread function, such as a power function, ${ }^{7}$ is approximated. In lateral modulation, 
the coherent superimposition of the steered beams performed in lateral modulation has a higher potential for realizing more accurate measurement of a displacement vector than the synthesis of the displacement vector using the accurately measured axial displacements performed with the MDSAM and the multiple transmitting method. ${ }^{3}$ However, note that for lateral modulation, MDSAM and the multiple transmitting method can also be used to obtain the steered beams. When using the multiple transmitting method for lateral modulation, plural transducers can also be used. Almost the same high lateral resolution can be obtained for the axial resolution. ${ }^{1}$

However, although with lateral modulation when the multiple transmitting method is used, a large transmitted ultrasound intensity can be realized by beamforming using plural ultrasound elements, when MDSAM is used, a small transmitted ultrasound intensity can be obtained using a single transmission element with a synthetic aperture method. Indeed, both in conventional beamforming with only an axial frequency and in lateral modulation beamforming, the use of a solo ultrasound transmission element decreases the echo signal-to-noise ratio at digitization. In conjunction with this, MDSAM may be affected by rapid target motion. However, when using the multiple transmitting method by transmitting plural beams at different times, the rapid target motion during multiple transmissions/receptions of ultrasound beams will definitely lead to deterioration in B-mode imaging and in the target tissue's motion measurement.

Thus, in this report, a new synthetic aperture method using a new virtual source ${ }^{8}$ is described. Because the synthetic aperture method performs a summation of random scattered echos, being different from the virtual source previously reported, ${ }^{9,10}$ the new virtual source will not always need to be placed at the focus position of a physical or effective aperture, ie, it can be placed in an arbitrary position. The new viewpoint will permit increases in the applications of a virtual source as discussed in this report. That is, the new virtual source can acquire a large transmitted ultrasound intensity and a high lateral resolution of synthesized echo data and, consequently, a robust synthetic aperture beamforming with respect to rapid target motion can be realized.

Originally, an ultrasound virtual source was utilized for obtaining a high lateral resolution and a large ultrasound transmission intensity with conventional B-mode imaging. ${ }^{9,10}$ The virtual source is set at a focus position of a physically large aperture, a synthesized aperture, or an individual element aperture ${ }^{9-11}$ and, in general, the virtual source location must be outside a region of interest. However, for a linear array type transducer, a virtual source array synthesized yields only the almost same lateral resolution as that directly obtained using the physical aperture synthesized. ${ }^{11}$ In order to increase the lateral resolution, the removal of diffraction effects of virtual source array elements is performed. ${ }^{12}$

Alternatively, with a newly developed virtual source using the new viewpoint, because the virtual source can also be set at an arbitrary position behind the ultrasound transducer, it is possible to examine regions adjacent to the transducer. This allows superficial tissues, such as skin, to be dealt with. Actually, such a beamforming has been performed since $1984 .{ }^{13,14}$ After the first application of beamforming to a linear-phased array imaging, ${ }^{14}$ a circular ring array aperture ${ }^{15}$ has also been used. A linear array type transducer has also been used by other groups. ${ }^{16-18}$ Initially, the non-physically set source was referred to as a virtual source or a virtual element. ${ }^{13,14,17}$ Applications to other type apertures have also been reported, eg, a small cylindrical aperture. $^{19}$

In our case, using the same new viewpoint, another virtual source can also be obtained, and is constructed using an ultrasound random scatter, ${ }^{2}$ a diffraction grating, or a hole. ${ }^{8}$ That is, an arbitrary position ahead of the physical array elements is also used as a virtual source position, eg, in a target tissue or in some material put between the physical aperture and the target.

Because both virtual sources obtained using the new viewpoint can be dealt with as a point acoustic source, it is also expected that the lateral resolution will be higher than those of the other virtual sources or physical apertures mentioned above. Thus, in this report, after describing the virtual sources summarized using the new viewpoint and the properties of such virtual sources, in agar phantom experiments, the limitations of accuracy and spatial resolution of the proposed virtual sources are confirmed for lateral modulation B-mode imaging, displacement vector/strain tensor measurements, and shear modulus reconstructions. Finally, discussions and conclusions are provided.

\section{New virtual sources}

As shown in Figure 1a, the setting of a virtual source behind the transducer array ${ }^{8,13-19}$ yields a larger transmitted ultrasound intensity by firing plural elements rather than a physical source as used with the classical synthetic aperture method. In this report, this virtual source is referred to as VS1. Alternatively, Figure 1b shows another previously reported virtual source ${ }^{9-11}$ with the virtual source set at the focus positions of a physically large aperture, a synthesized aperture, or an individual element aperture. 
A

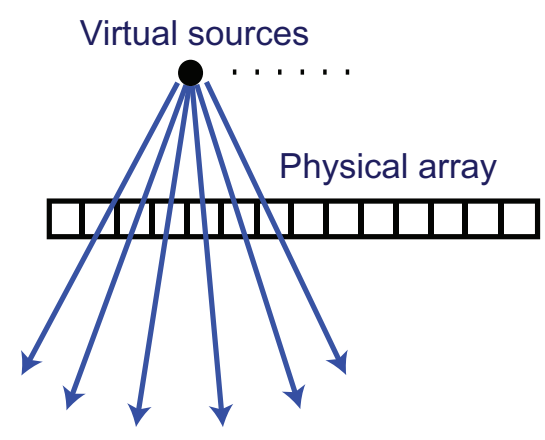

C

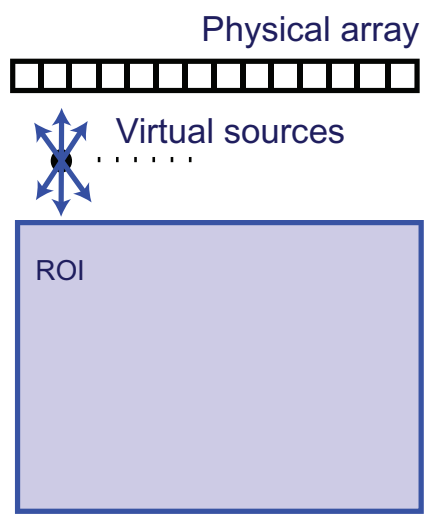

D

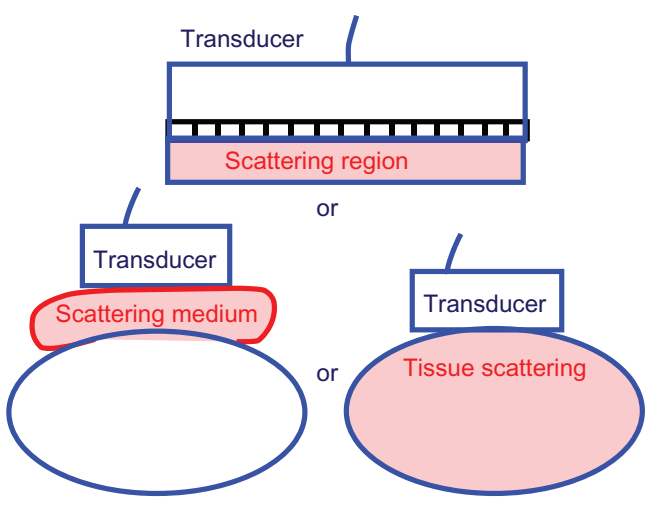

B

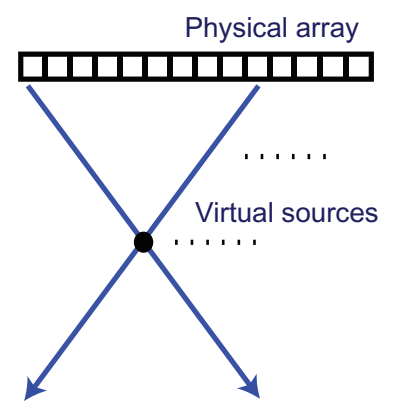

E
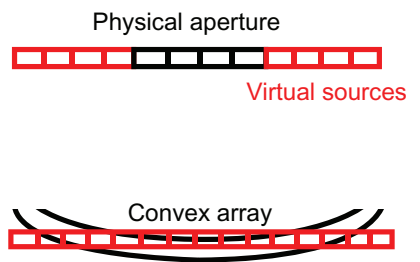

Virtual sources

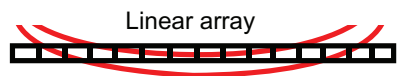

Virtual convex array

Regeneration of vision field, beam direction etc.

Figure I A) Virtual sources (VSI) set behind physical array elements for gaining a transmitted ultrasound intensity without removing a superficial region from the region of interest (ie, without shortening the axial length of the region of interest); ${ }^{13-19} \mathrm{~B}$ ) Virtual sources ${ }^{-12}$ set at the focus position of a physical aperture; C) Virtual sources (VS2) of which position is independent of physical apertures (ie, an arbitrary position ahead of physical array elements); ${ }^{8} \mathbf{D}$ ) those realized by regular or random scatter material installed in transducers, by a scatter medium put between the transducer and target, or random scattering in a target tissue; ${ }^{8} \mathbf{E}$ ) those (or receivers) realized in null spaces beside the short physical array aperture to obtain a large width for the region of interest and to obtain an arbitrarily shaped field of vision regardless of physical aperture geometry (eg, convex, linear). ${ }^{8}$

When using an array aperture, both the virtual sources gain transmitted ultrasound intensity from using plural transmission elements rather than a physical source (ie, one element) for the classical synthetic aperture method. That is, both the virtual sources yield a larger echo signal-to-noise ratio. In addition, although the virtual source focus position must be removed from the region of interest, VS1 does not decrease the axial length of the region of interest, but increases the extent of a region of interest. ${ }^{18}$
In this report, VS1 is dealt with as a point acoustic source ${ }^{8}$ similarly to that originally reported. ${ }^{13-19}$ Then, for the use of the synthetic aperture method, a spherical wave is considered as a generated wave. In this case, ultrasound to be transmitted from or received by the respective physical elements is weighted by decay weights which are determined by the reciprocal of the distance between the virtual source and the respective physical elements (see Figure 1a). Otherwise, the decay weights can also be determined more strictly by using the distances 
between the point of interest and the physical elements and between the point of interest and the virtual source. Using the superposition theorem, VS1 can also be used for real-time beamforming for imaging and measurements, ie, so-called dynamic focusing and dynamic apodization. For VS1, other types of virtual sources used not as a point acoustic source but as a source having a finite aperture size are discussed in the final section, because decreases in lateral resolution were obtained in the agar phantom experiments, although the virtual source with an infinitesimal size was used.

Another obtained virtual source (VS2) ${ }^{8}$ using the same new viewpoint is also realized, ie, by using ultrasound random scatters or regularly aligned scatters in a scatter medium (material). It can also be realized independently of the focus position of a physical aperture, but in the neighborhood of the physical aperture as shown in Figure 1c. These can be realized by installing the ultrasound scattering medium in the transducer, between the transducer and the target body, or in the target medium (Figure 1d). ${ }^{2}$ Because a summation of random scattering echos is used with the synthetic aperture method, a virtual source may not always be placed at the focus position of a physically large aperture, a synthesized aperture, or individual element apertures. ${ }^{9,10}$ Thus, VS2 is set in an arbitrary position with no regard for the physical focus positions.

In this report, the proposed VS2 is also dealt with as a point acoustic source. However, being different from VS1, only the synthetic aperture method can be applied to echo data set physically obtained. For the synthetic aperture method, the intensities of ultrasound received by the respective physical elements is corrected using decay weights which are determined by using the distances between the point of interest and the physical element and between the point of interest and the virtual source (see Figure 1c). In the agar phantom experiments, VS2 achieved significantly higher lateral resolutions than the physical array source. To obtain such a high lateral resolution, in general, a smaller physical array source and a smaller pitch must be used, but leading to a low echo signal-to-noise ratio due to their physical and electrical limitations, ie, energy conversion efficiency. Thus, VS2 also allows mitigations of the limitations for using smaller ultrasound array elements and a smaller pitch, such that a high echo signal-to-noise ratio can also be obtained. That is, VS2 allows the use of a larger physical array source for obtaining the same lateral resolution. VS2 can also be realized by using a proper diffraction grating or a hole like a point acoustic source. In the final section, for VS2, such other types of virtual sources are also discussed.

The new viewpoint used by VS2, as well as VS1, will further increase the applications for a virtual source. For instance, virtual sources (or receivers) can be realized in null spaces beside the short physical array aperture by searching for the corresponding echo data in an acquired echo data set (Figure 1e). Thus, the lateral width as well as the axial length of a field of vision will also increase. When a transducer has a small physical array aperture, lateral modulation cannot deal with deeply situated tissues, ${ }^{3}$ but new virtual sources will be used to mitigate such limitations. Alternatively, an arbitrary shaped field of vision can also be obtained regardless of the physical aperture geometry (see Figure 1e for convex, linear, etc).

\section{Agar phantom experiments}

Experiments were performed using the same agar phantom which was described previously. ${ }^{2}$ The target agar phantom (40 [axial, x] $\times 96$ (lateral, y) $\times 40$ (elevation) $\mathrm{mm}^{3}$ ) had a central circular cylindrical inclusion (diameter $10.0 \mathrm{~mm}$, depth $19.0 \mathrm{~mm}$ ) with a shear modulus different from that of the surrounding region, and shear moduli were 2.63 and $0.80 \times 10^{6} \mathrm{~N} /$ $\mathrm{m}^{2}$ in the inclusion and in the surrounding regions, respectively (ie, a relative shear modulus of 3.29). The concentrations of the agar (Wako Pure Chemical Industries, Ltd., agar powder 010-15815, Osaka, Japan) in the cylindrical inclusion and in the surrounding region were $6.0 \%$ and $3.0 \%$, respectively. To control ultrasound attenuation, graphite powder (graphite powder 070-01325, concentration 3.0\%; Wako Pure Chemical Industries, Ltd., Osaka, Japan) was also added. The shear moduli were measured by performing loading tests on block phantoms with the same agar concentrations.

Manually, the phantom was compressed by $2.0 \mathrm{~mm}$ in the lateral direction. The contact surfaces of the linear array type transducer $(7.5 \mathrm{MHz}, 0.2 \mathrm{~mm}$ ultrasound element pitch, $2.4 \mathrm{~mm}$ depth for an acoustic lens involving matching layers) and the phantom were separated by less than $0.3 \mathrm{~mm}$ by immersing them in water in a tank, and a sponge was put under the phantom to allow the phantom to elongate in the axial direction through uniform lateral compression from the right-hand side using a large plate. The left surface was fixed to a wall. A rectangular region of interest 13.7 (axial, $\mathrm{x}) \times 13.2$ (lateral, y) $\mathrm{mm}$ was centered on the inclusion (depth from 12.2 to $25.9 \mathrm{~mm}$ ).

As shown in Figure 2, the depth of a lateral line in which point virtual sources were set changed from $-19.0 \mathrm{~mm}$ to $9.0 \mathrm{~mm}$ with respect to the linear array surface $(0.0 \mathrm{~mm})$. The minus position corresponds to a position behind the array surface (ie, the position of VS1), whereas the plus position corresponds to a position in front of the array surface (ie, the position of VS2 in the acoustic lens or target phantom). 


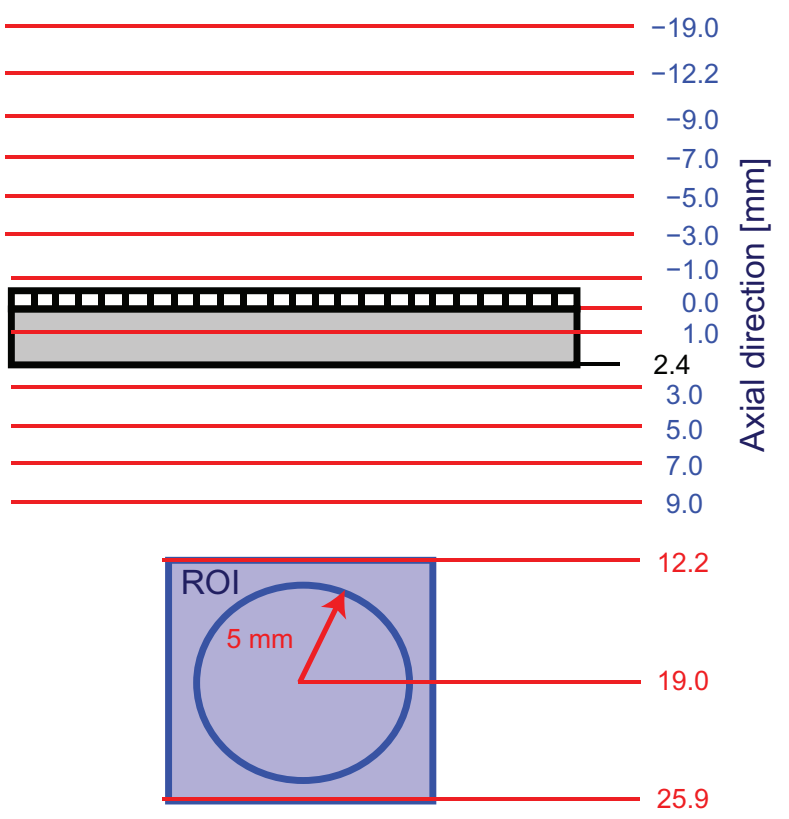

Figure 2 The depth positions of lines arranged by virtual sources with respect to a physical array realized by ultrasound elements $(0.0 \mathrm{~mm})$ and a region of interest ranging from I 2.2 to $25.9 \mathrm{~mm}$, ie, for VSI, $-19.0,-12.2,-9.0,-7.0,-5.0,-3.0$, and $-1.0 \mathrm{~mm}$, and for VS2, I.0, 3.0, 5.0, 7.0, and $9.0 \mathrm{~mm}$. The shaded region attached to the physical array is an acoustic lens involving matching layers with a $2.4 \mathrm{~mm}$ depth.

With lateral modulation, parabolic modulation was performed. ${ }^{2}$ This is, Gaussian functions which express an apodization function obtained by a Fraunhofer approximation of a Gaussian-type point spread function were changed by parabolic functions so that the transmitted ultrasound intensity is invariant. Using the same ultrasound intensity, use of the parabolic functions yields a higher echo signal-tonoise ratio than that of the Gaussian functions. As described previously, ${ }^{2}$ the lateral modulation frequency was $3.75 \mathrm{MHz}$ and the standard deviation (SD) for the Gaussian type point spread function was $0.4 \mathrm{~mm}$. For both transmission and reception, a dynamic spherical focusing was performed.
For two-dimensional (2-D) displacement vector measurements, the 2-D autocorrelation method $^{3}$ was used with a moving-average window size of $0.54 \times 0.55 \mathrm{~mm}$. For a coarse measurement, to prevent multidimensional autocorrelation method from being subjected to aliasing in its evaluation of the instantaneous phase due to the large lateral displacement (maximum, $2 \mathrm{~mm}$ ), a 2-D cross-correlation method was used (local window size, $0.54 \times 2.15 \mathrm{~mm}$ ). That is, the local region size used was made small during phase matching. ${ }^{3,4}$ The 2-D strain tensor components were obtained using a differential filter ${ }^{20}$ with a cutoff frequency of $0.89 \mathrm{~mm}^{-1}$. For 2-D reconstruction, a method using a typical Poisson ratio (0.5) was used under a 2-D stress condition. ${ }^{20}$ Because the phantom was predominantly deformed in the lateral direction, the reference region was set on the right borderline of the region of interest (a reference shear modulus of 1.0).

Figure 3 shows the B-mode image and the images of lateral and axial displacements (ie, dy, dx), a relative shear modulus reconstruction, and lateral, axial, and shear strains (ie, $\varepsilon_{\mathrm{yy}}, \varepsilon_{\mathrm{xx}}$, and $\varepsilon_{\mathrm{xy}}$ ) obtained for physical sources (ie, array position, $0.0 \mathrm{~mm}$ depth) and for VS1 at depths of -1.0,-3.0, $-5.0,-7.0,-9.0,-12.2$, and $-19.0 \mathrm{~mm}$. Figure 4 shows the images obtained for VS2 at depths of $1.0 \mathrm{~mm}$ (ie, in the acoustic lens) and 3.0, 5.0, 7.0, and $9.0 \mathrm{~mm}$ (in the phantom). As shown in all of the B-mode images (Figures 3 and 4), a lateral modulation is achieved. However, the specular reflection circled in Figure $3 \mathrm{a}$ (ie, physical sources used) cannot be confirmed from the images obtained using virtual sources positioned outside the depths in the range of $\pm 3 \mathrm{~mm}$.

For the synthesized rf-echo data that correspond to Figures $3 \mathrm{a}-3 \mathrm{~h}$ and $4 \mathrm{a}-4 \mathrm{e}$, the lateral resolutions at a central depth line (19.0 $\mathrm{mm}$ depth), and upper (12.2 mm)

A $0 \mathrm{~mm}$

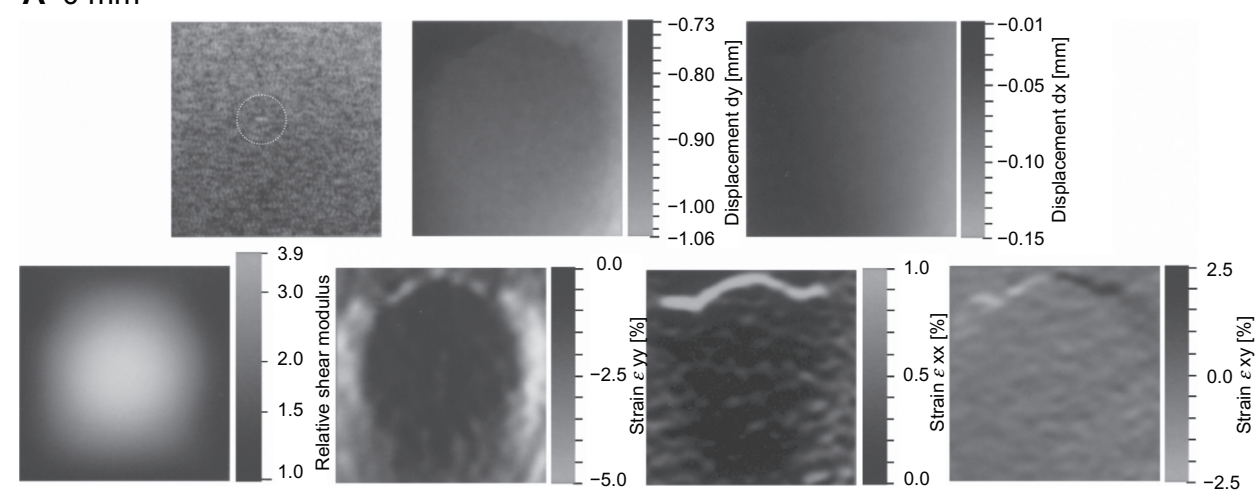

Figure 3 (Continued) 
B $-1 \mathrm{~mm}$

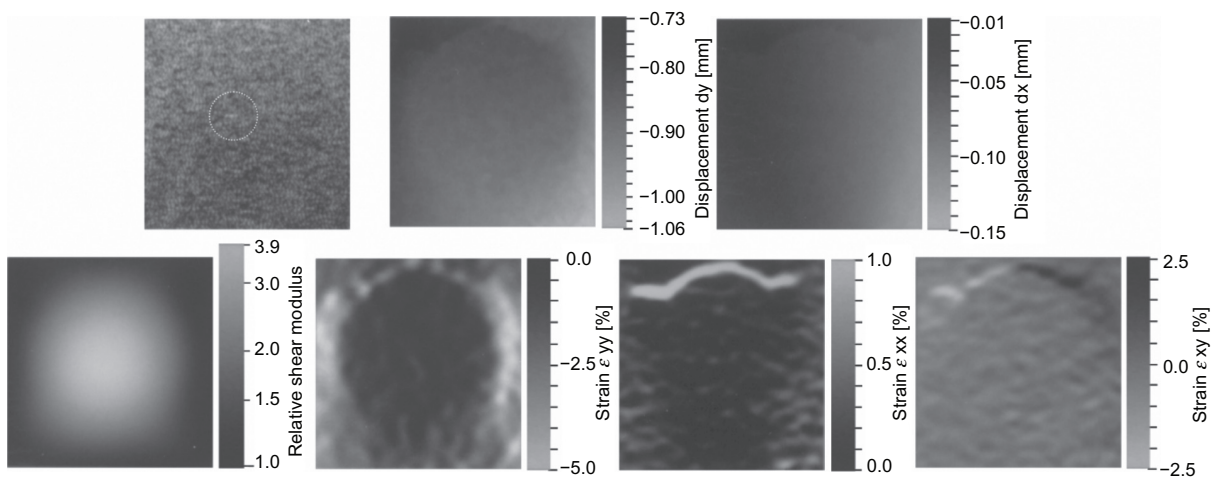

C $-3 \mathrm{~mm}$
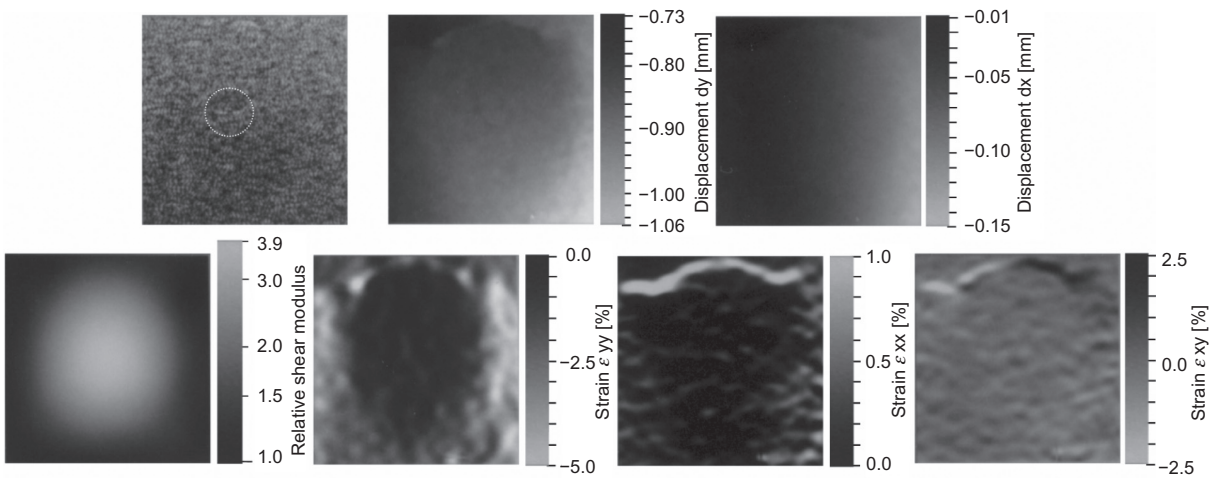

D $-5 \mathrm{~mm}$

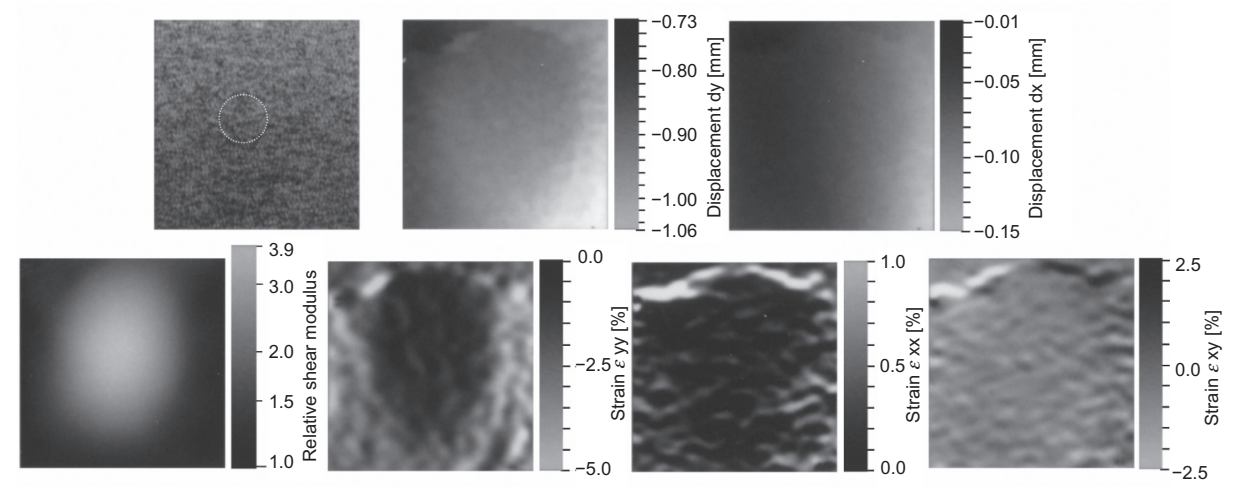

E $-7 \mathrm{~mm}$

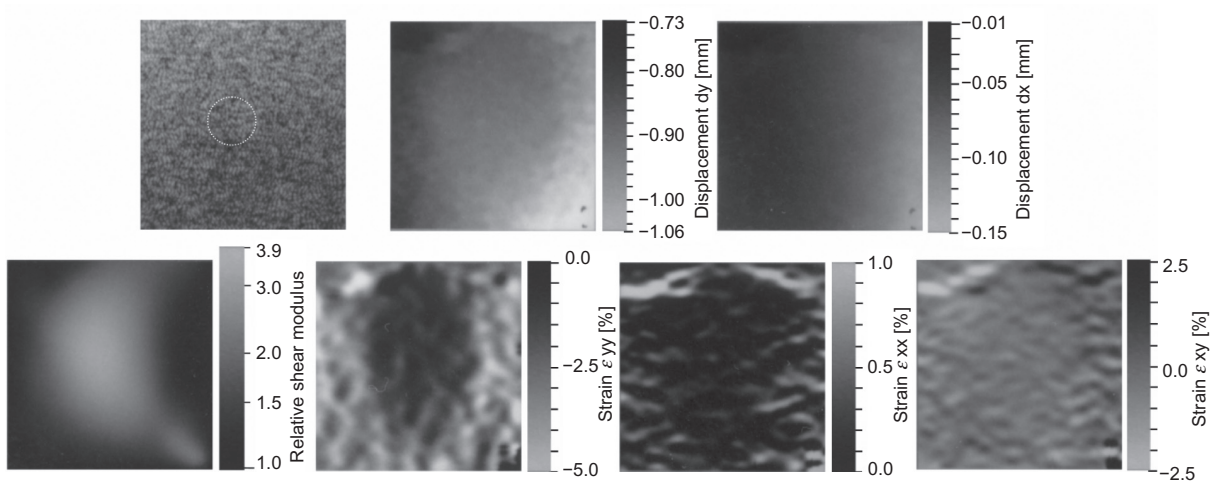

Figure 3 (Continued) 


\section{F $-9 \mathrm{~mm}$}
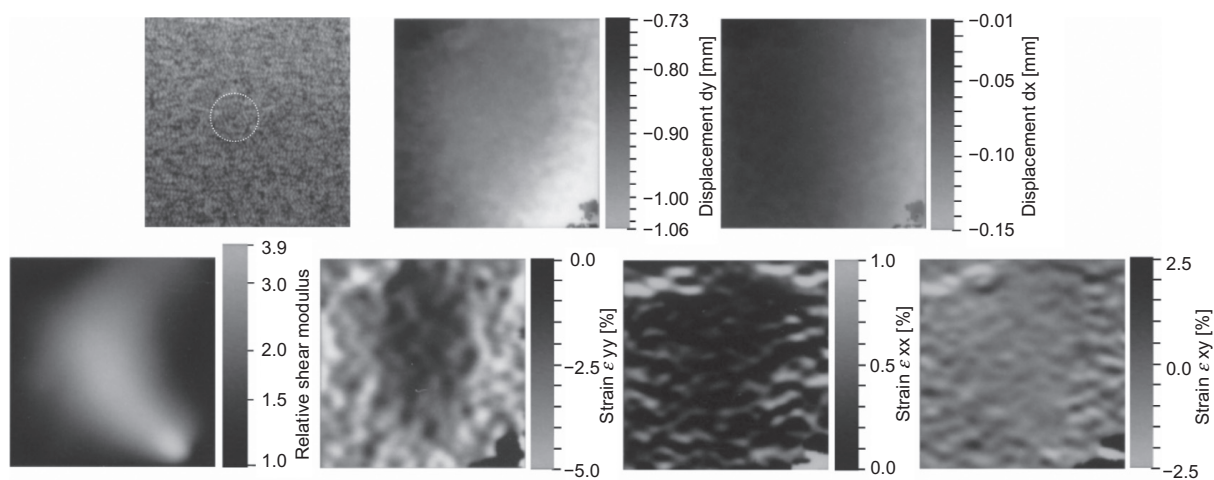

G $-12.2 \mathrm{~mm}$

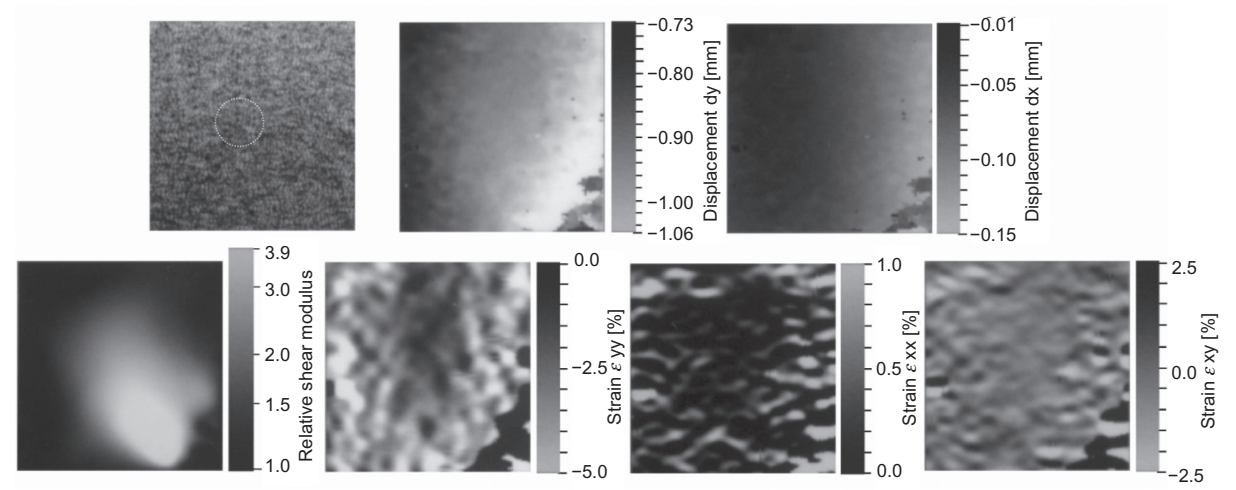

H $-19 \mathrm{~mm}$

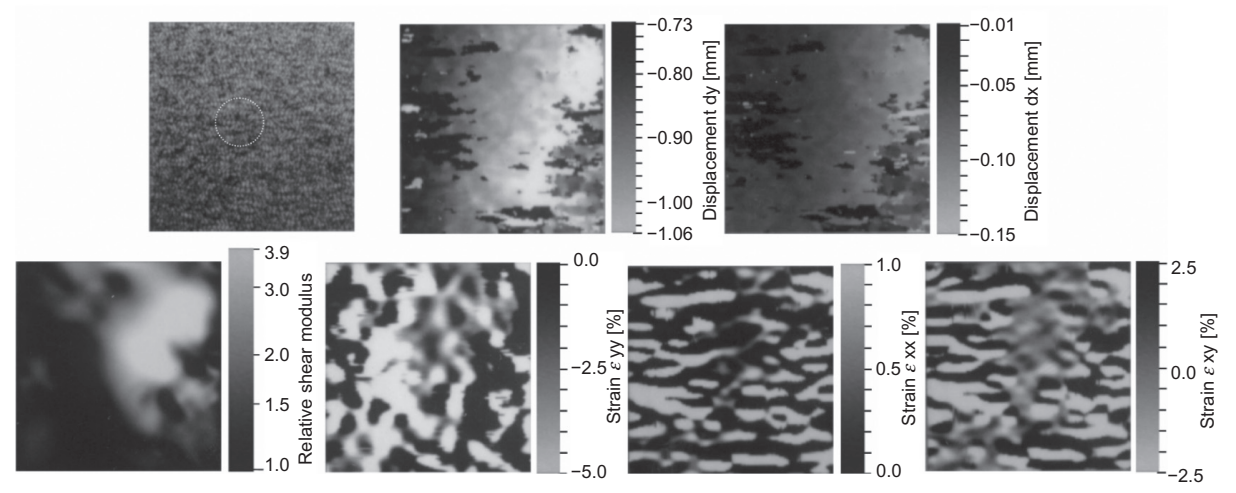

Figure 3 B-mode image and measured lateral and axial displacements (ie, dy and dx) using lateral parabolic modulation and the two-dimensional (2-D) autocorrelation method, from which relative shear modulus reconstruction (2-D stress assumption), and lateral, axial and shear strains (ie, $\varepsilon_{y y}, \varepsilon_{x x}$ and $\varepsilon_{x y}$ ) were obtained. Those obtained for

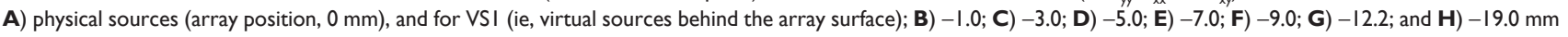
depths. The position of specular echo detected in (a) to (c) is circled.

and lower $(25.9 \mathrm{~mm})$ borderlines of the region of interest are evaluated using a lateral shape of 2-D autocorrelation function. Figures 5a-5c show respectively for physical sources ( $0 \mathrm{~mm}$ depth), VS1 with a depth $-3 \mathrm{~mm}$ and VS2 with a depth $+3 \mathrm{~mm}$ for the lateral shapes. As shown, side lobes of the autocorrelation functions attenuate and have lower frequency oscillations. The lateral widths of main lobes of the autocorrelation functions reveal that for VS1 and VS2, as well as for the physical sources, the synthetic aperture yields the almost uniform lateral resolutions with respect to the imaging depth (ie, 12.2, 19.0, and $25.9 \mathrm{~mm}$ ). Specifically, however, the lateral resolution slightly decreases for the imaging of a deeper line, particularly for VS1 (Figure 5b). Interestingly, although the lateral resolutions for VS1 are lower than those for the physical sources, those of VS2 are higher. For upper $(12.2 \mathrm{~mm})$ and lower $(25.9 \mathrm{~mm})$ borderlines of the region of 


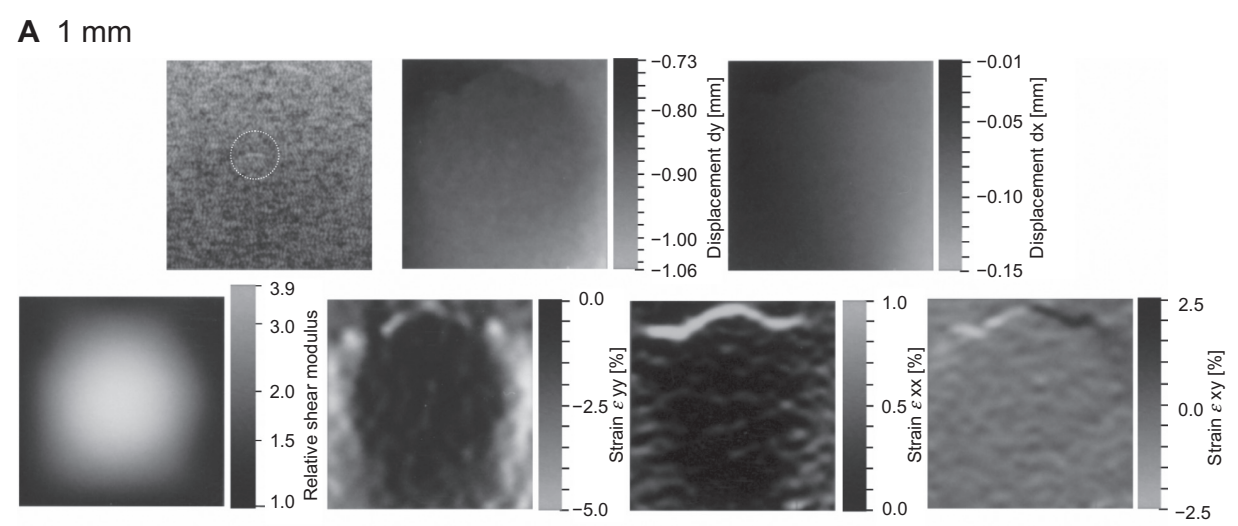

B $3 \mathrm{~mm}$

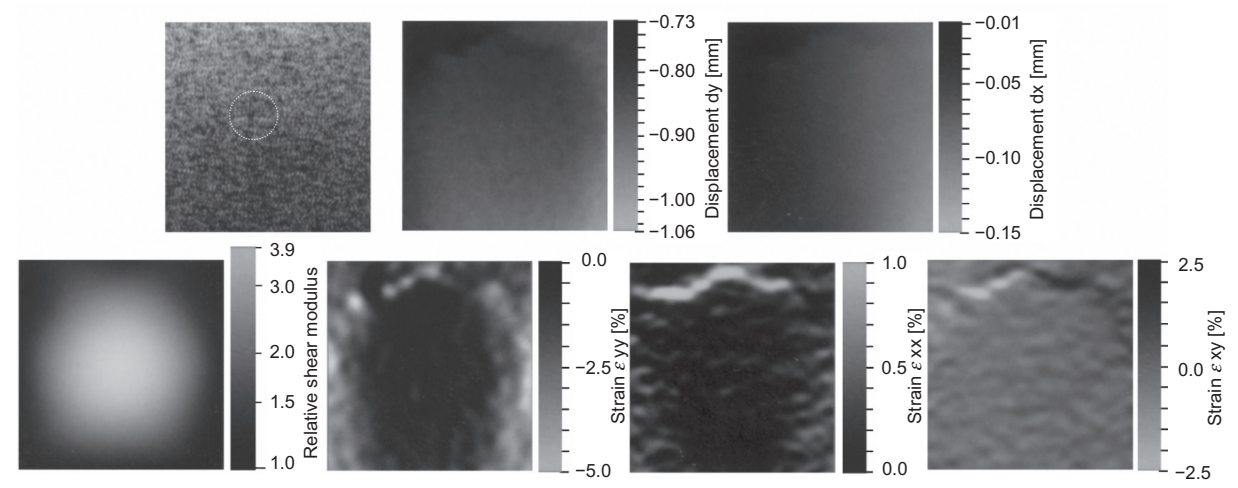

C $5 \mathrm{~mm}$

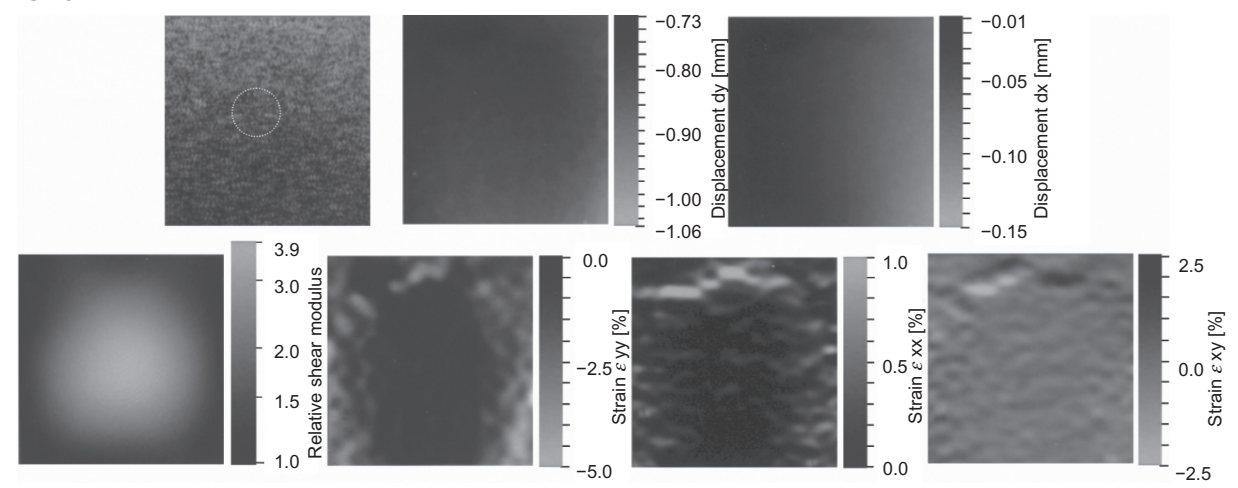

D $7 \mathrm{~mm}$

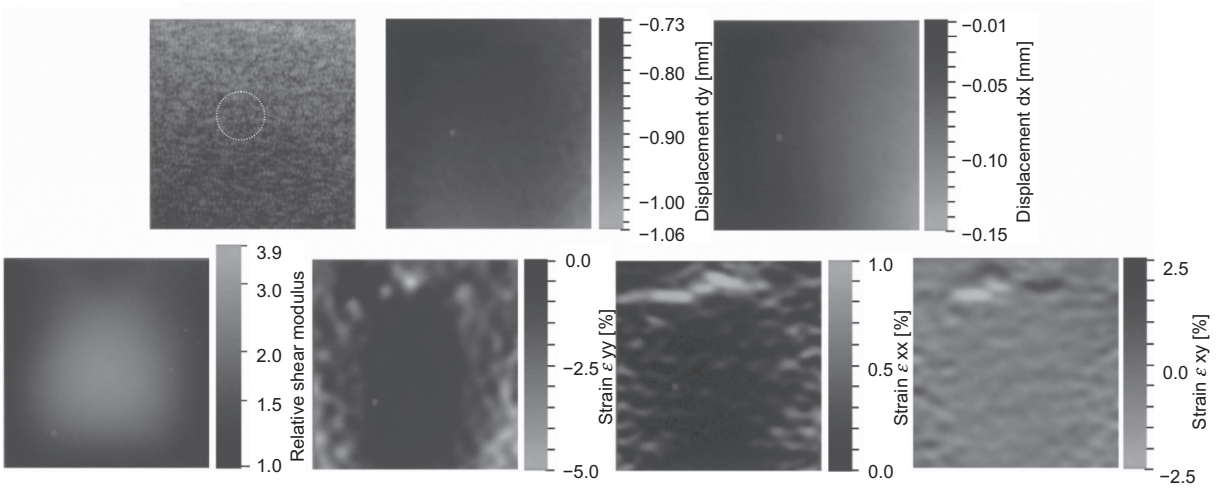

Figure 4 (Continued) 


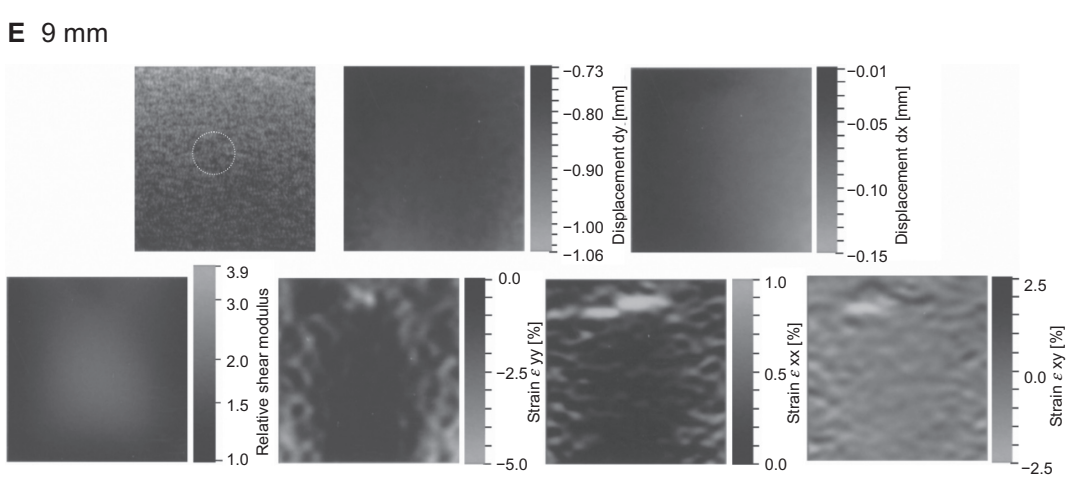

Figure 4 The same images as those in Figure 3 (see Figure 3 legend), but obtained for VS2 (ie, virtual sources ahead of the array position at $0.0 \mathrm{~mm}$ depth) A) $1.0 \mathrm{~mm}$ depth (ie, in an acoustic lens); B) 3.0; C) 5.0; D) 7.0; and E) $9.0 \mathrm{~mm}$ depths (ie, in a phantom). Position of specular echo detected in (A) and (B) is circled.

A

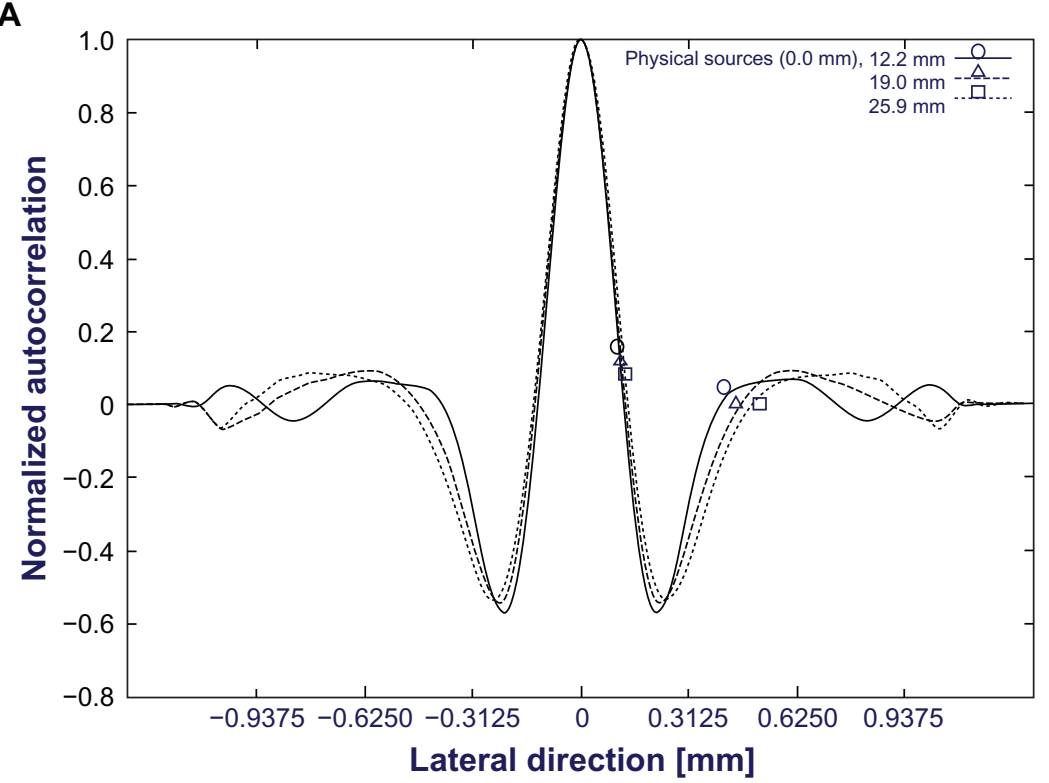

B

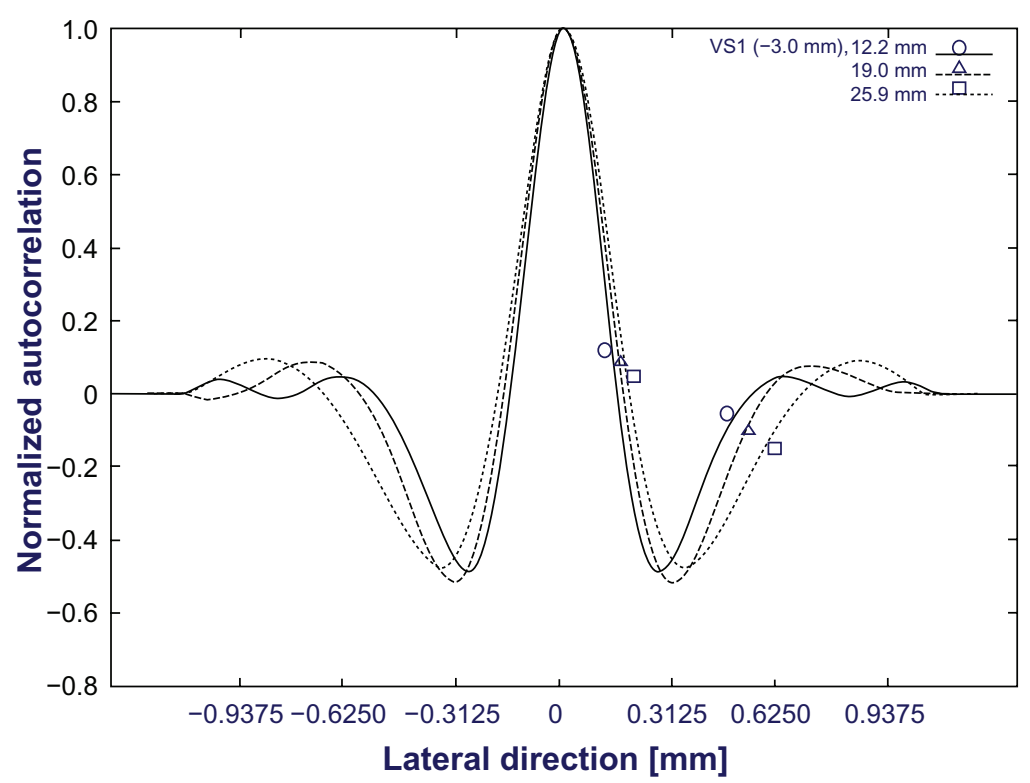

Figure 5 (Continued) 
C

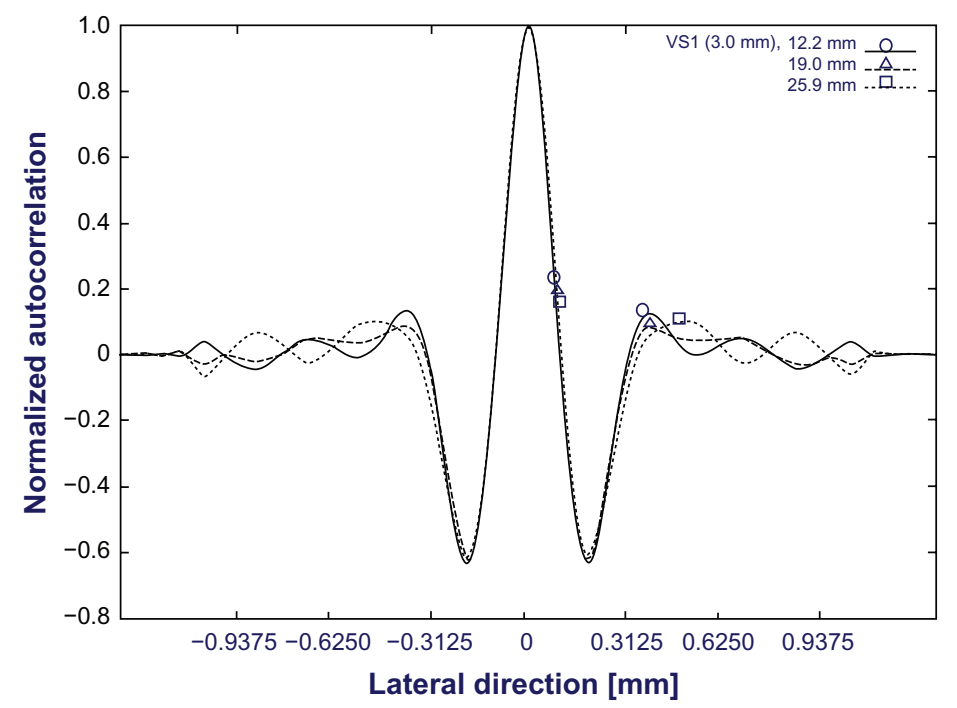

Figure 5 Lateral shapes of normalized two dimensional autocorrelation functions obtained at imaging depths of I2.2 (upper borderline of region of interest), 19.0 (central depth line), and 25.9 (lower borderline) $\mathrm{mm}$ for (A) physical sources (array position, $0 \mathrm{~mm}$ ); (B) VSI with $-3.0 \mathrm{~mm}$ depth; and (C) VS2 with $3.0 \mathrm{~mm}$ depth.

interest, Figures $6 \mathrm{a}-6 \mathrm{~d}$ respectively show the lateral shapes of the autocorrelation functions for VS1 $(-1.0$ to $-19.0 \mathrm{~mm})$ and VS2 (1.0 to $9.0 \mathrm{~mm})$ with respect to that of the physical sources $(0.0 \mathrm{~mm})$. For VS1, with the distance between VS1 and the physical sources increasing, the low lateral resolution increases up to that of the physical aperture, although the position to $-3.0 \mathrm{~mm}$ depth is meaningful as confirmed above. That is, VS1 yielded lower lateral resolutions than the physical aperture. Alternatively, for VS2, with the position approaching the physical sources, the lateral resolution increases and, remarkably, VS2 up to $9.0 \mathrm{~mm}$ depth achieves higher lateral resolutions than that of the physical sources. Thus, VS1 and VS2 should be positioned far from the physical sources and in the neighborhood of the physical sources, respectively, and VS2 using a point acoustic source model achieves higher lateral resolutions than that of the physical sources, although VS1 yields lower lateral resolutions.

Figure 7 shows the means and SDs of lateral, axial, and shear strains, as well as shear moduli evaluated in the central $5 \mathrm{~mm}$ square region in the inclusion. In the depth range of $\pm 3 \mathrm{~mm}$, accurate shear modulus reconstructions were obtained. In addition to the lower lateral resolutions obtained, VS1

A

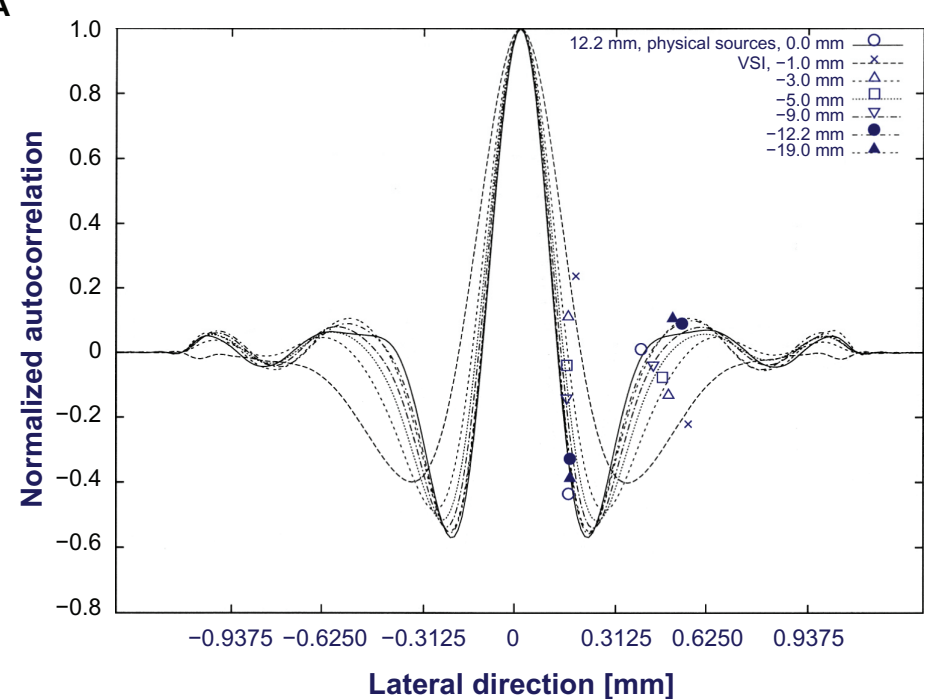

Figure 6 (Continued) 
B

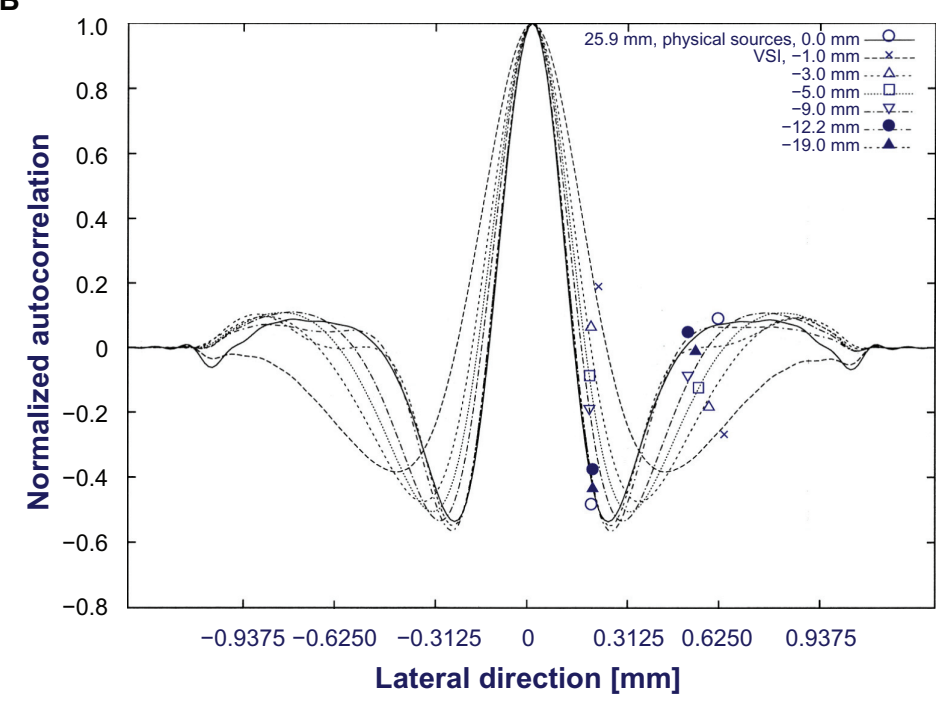

C

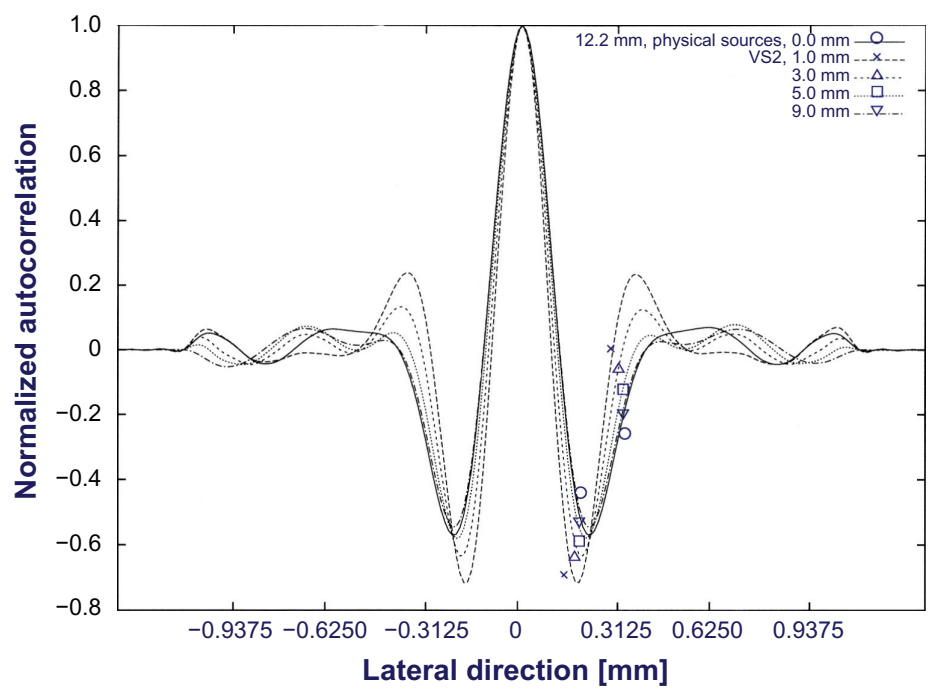

D

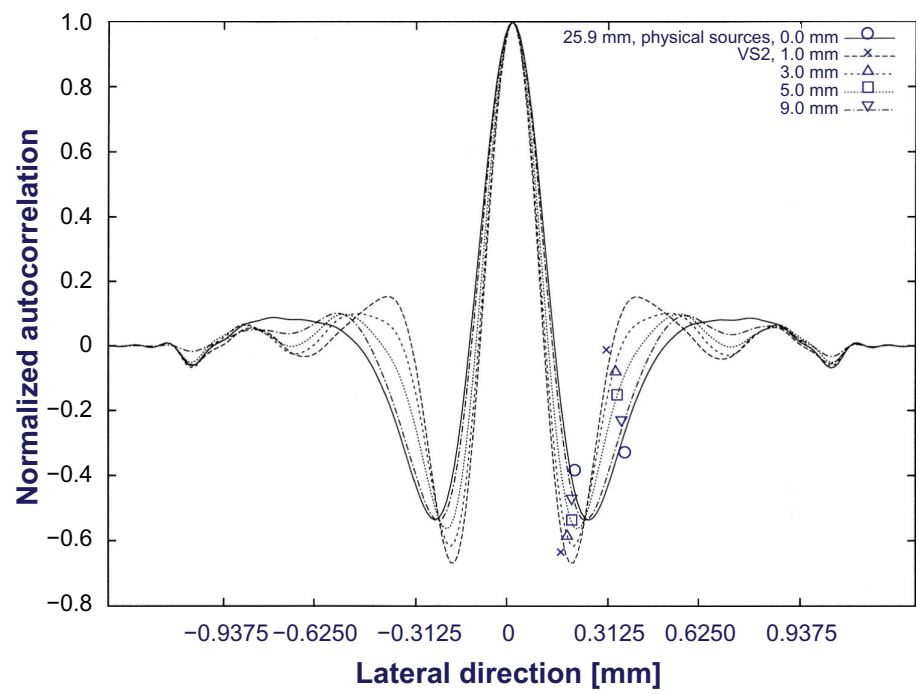

Figure 6 Lateral shapes of normalized two-dimensional autocorrelation functions obtained at imaging depths of 12.2 (upper borderline of region of interest) and 25.9 (lower borderline) $\mathrm{mm}$ for physical sources (array position, $0 \mathrm{~mm}$ ), VSI with $-1.0,-3.0,-5.0,-9.0,-12.2 \mathrm{~mm}$ and $-19.0 \mathrm{~mm}$ depths [(A) $12.2 \mathrm{~mm}$ and (B) $25.9 \mathrm{~mm}]$ and VS2 with I.0, 3.0, 5.0 and $9.0 \mathrm{~mm}$ depths [(C) $12.2 \mathrm{~mm}$ and (D) $25.9 \mathrm{~mm}]$. 
A

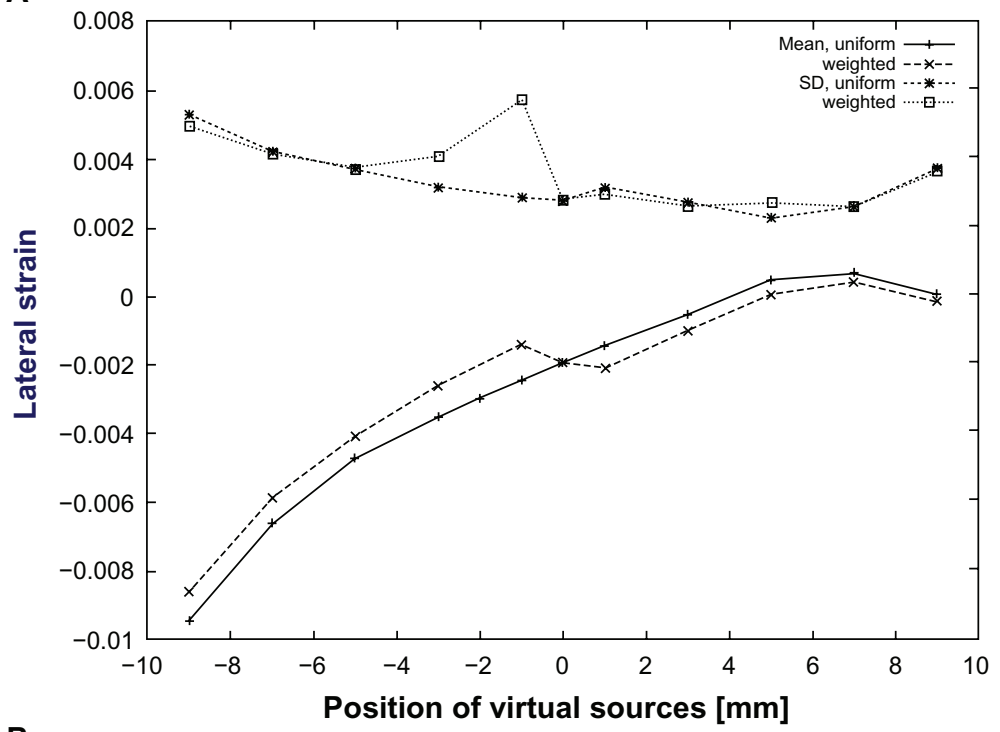

B

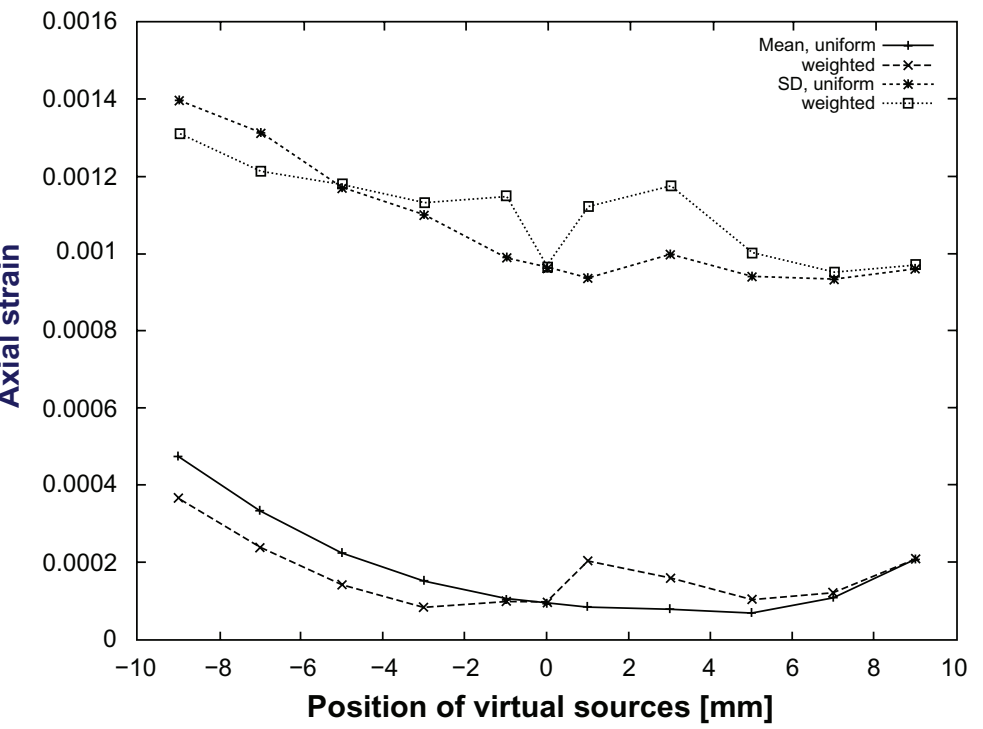

C

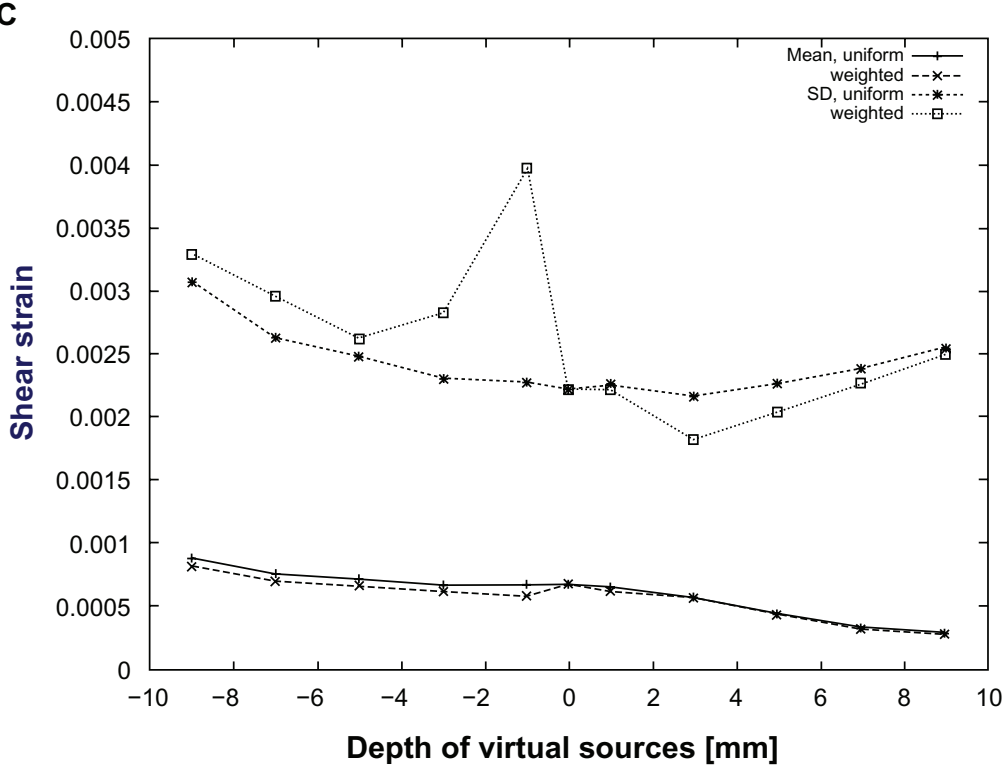

Figure 7 (Continued) 


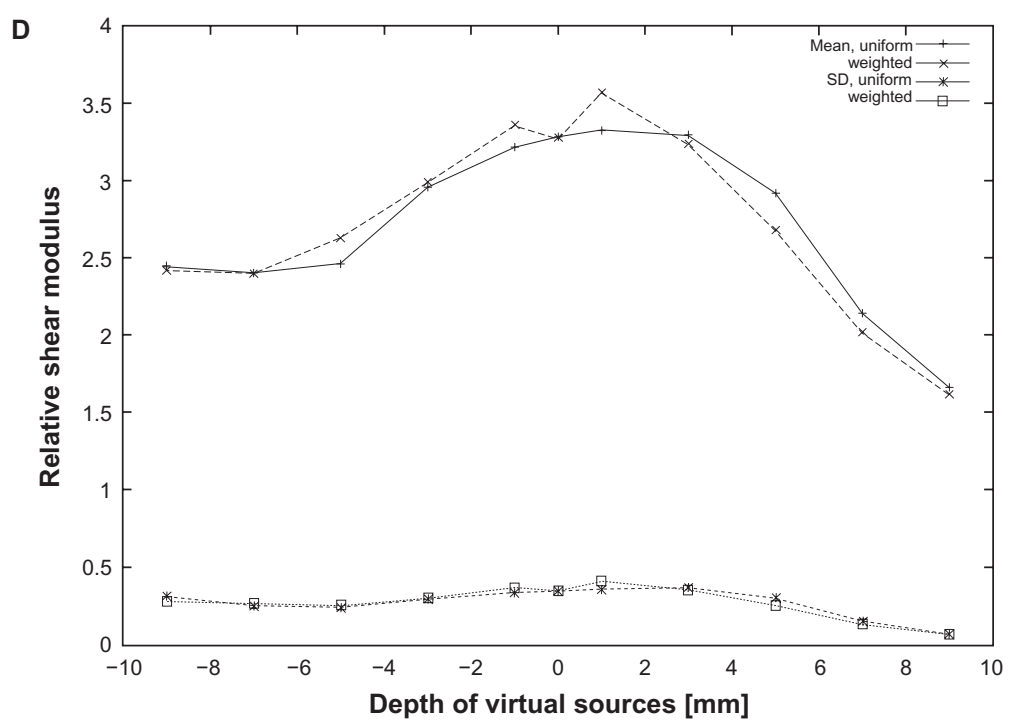

Figure 7 Means and standard deviations of measured (A) lateral; (B) axial and (C) shear strains; and (D) reconstructed relative shear moduli versus axial depth for virtual sources for use and no use of decay weights for the multidirectional synthetic aperture method.

yields larger SDs than VS2, particularly when the distance between the positions of VS1 and physical sources increases. This occurred because the far field from VS1 was directly generated by ultrasound transmissions from the large size ultrasound elements with a large pitch. That is, a phase error for a spherical wave was generated. In Figure 7, those obtained by the use of decay weights for a spherical wave are also shown. Although there are no large differences between the results obtained with or without the use of the decay weights, the use of weights slightly increased instabilities in the measurements and reconstruction, particularly with VS1 approaching the physical sources (Figures 7a-7c). This also occurred because the ultrasound element size was large and the element pitch was coarse, and the echo data acquisition for synthetic aperture method was performed using the same transmitted ultrasound intensity from the respective physical elements. That is, noise in the acquired echo data for the synthetic aperture method was also weighted along with the original echo signals by the decay weights. On the contrary, the distance between VS1 and the physical sources increasing, the use of weights yielded more stable measurements and reconstruction (Figures $7 \mathrm{a}$ and $7 \mathrm{~b}$ ).

Regarding VS2, when the decay weights was not used, SDs were almost constant, whereas the use of the weights led to different SDs from those with no use of weights, particularly, when the distance between VS2 and physical sources was small (Figure $7 \mathrm{~b}$ ). This is also due to the improper physical sources used.

\section{Discussion}

In this report, the newly developed virtual sources VS1 and VS2, along with preliminary experimental results, were presented for lateral modulation. Agar phantom experiments were performed. Summarizing, VS1 permits an increase in the transmitted ultrasound intensity using plural transmission ultrasound elements without decreasing, but instead increasing, the size of the region of interest (ie, the virtual source is set behind the physical array elements). VS2 uses an arbitrary position ahead of the physical array elements as a virtual source position. When performing a synthetic aperture beamforming, VS2 increases the lateral resolution as well as echo signal-to-noise ratio, and yield B-mode imaging and tissue displacement/strain measurements along with associated applications (eg, shear modulus reconstructions) with a high degree of accuracy. With VS2, a random scattering medium (material) can also be used for virtual sources. Remarkably, VS2 using a point acoustic source model yielded higher lateral resolutions than that of physical element sources. Specifically, VS2 yielded a higher lateral resolution than a synthesized physical aperture and other virtual sources reported.9-11,13-19 The high lateral resolution achieved is uniform regardless of the position depth. VS2 also mitigates the physical and electrical limitations of using smaller ultrasound array elements and a smaller pitch, such that a high echo signal-to-noise ratio as well as a high lateral resolution is obtained. Regarding VS1, the high lateral resolution achievable will be examined by using a proper transducer. Although not shown in this report, the same effective VS1 and VS2 were also obtained for a conventional beamforming, ie, with no lateral modulation. Such results will be reported elsewhere.

However, as shown in the agar phantom experiments, the large range of depths which can be used for virtual 
source positions could not be obtained with the ultrasound transducer which was used, ie, only $\pm 3 \mathrm{~mm}$ with an ultrasound element pitch of $0.2 \mathrm{~mm}$ and ultrasound frequency $7.5 \mathrm{MHz}$. Moreover, the use of decay weights for a spherical wave increases instabilities in the displacement vector/strain tensor measurements and shear modulus reconstructions slightly, particularly when virtual sources are approaching the physical sources. To cope with these problems, smaller ultrasound elements with a smaller pitch will also be used in the near future. However, with increasing distance between the virtual and the physical sources, the use of weights yielded more stable measurements and reconstruction. Because the newly developed virtual sources will also mitigate problems involving rapid motion, dynamic experiments will also be conducted.

The aforementioned additional applications of these proposed virtual sources will also be enabled by considering the results obtained in preliminary experiments. That is, an arbitrarily shaped field of vision can be obtained regardless of the aperture geometry. Placing virtual sources (or receivers) in null spaces outside of the short physical array aperture will also increase the axial and lateral lengths of the field of vision. This should be particularly effective for beam steering as well as lateral modulation. The virtual sources will also be effective for a microscope, various types of transducers, and other beamformings, including various conventional beamformings, multiple transmitting methods, and MDSAM.

In addition, for VS1 and VS2, experiments will also be conducted to examine the use of another virtual source that is not a point acoustic source; for instance, one having a finite aperture size. That is, decay weights will be calculated analytically or numerically. Particularly, for VS2, regarding the scattering angle versus the ultrasound penetration, the geometry and acoustic impedance of scatter and media, and some regular, irregular, or random alignment or distribution of scatters, will also be examined in conjunction with a physical source. Realization of the proper diffraction grating should also be our research target. Moreover, the optimization method for determining the beamforming parameters, such as an apodization function involving delays, ${ }^{6}$ will also be used to set the position and intensity of virtual sources. Utilizing other concepts, a completely different virtual source can also be realized. ${ }^{21}$ This virtual source will be constructed with a mirror setting from a steered beam in a spatial domain, or in a single quadrant or octant spectra in a frequency domain. Thus, in addition to these proposed virtual sources, applications of these other virtual sources will also be reported.

\section{Disclosure}

The authors report no conflicts of interests in this work.

\section{References}

1. Sumi C. Beamforming for realizing designed point spread function. Proc IEEE Ultrasonics Symp. 2007;1557-1562.

2. Sumi C, Noro T, Tanuma A. Effective lateral modulations with applications to shear modulus reconstruction using displacement vector measurement. IEEE Trans Ultrason Ferroelect Freq Contr. 2008;55: 2607-2625.

3. Sumi C. Displacement vector measurement using instantaneous ultrasound signal phase - Multidimensional autocorrelation and Doppler methods. IEEE Trans Ultrason Ferroelect Freq Contr. 2008; $55: 24-43$.

4. Sumi C, Suzuki A, Nakayama K. Phantom experiment on estimation of shear modulus distribution in soft tissue from ultrasonic measurement of displacement vector field. IEICE Transactions on Fundamentals. 1995;E78-A:1655-1664.

5. Sumi C. Usefulness of ultrasonic strain measurement-based shear modulus reconstruction for diagnosis and thermal treatment. IEEE Trans Ultrason, Ferroelect Freq Contr. 2005;52:1670-1689.

6. Sumi C, Komiya Y, Uga S. A demonstration of optimal apodization determination for proper lateral modulation. Jpn J Appl Phys. 2009; 48(7B):07GJ06.

7. Sumi C, Shimizu K, Matsui N. Proper analytic point spread function for lateral modulation. Jpn J Appl Phys. 2010;49(7B):07HF07.

8. Sumi C, Uga S, Komiya Y. Lateral modulation - Use of virtual source (11th report). Proceedings of the 2009 Spring Meet. Acoustical Society of Japan. Tokyo; 2009;1381-1382. Japanese.

9. Passmann C, Ermert H. A 100-MHz ultrasound imaging system for dermatologic and ophthalmologic diagnostics. IEEE Trans Ultrason Ferroelect Freq Contr. 1996;43:545-552.

10. Frazier $\mathrm{CH}, \mathrm{O}$ 'Beien WD Jr. Synthetic aperture techniques with a virtual source element. IEEE Trans Ultrason Ferroelect Freq Contr. 1998; 45:196-207.

11. Bae MH, Jeong MK. A study of synthetic-aperture imaging with virtual source elements in B-mode ultrasound imaging systems. IEEE Trans Ultrason Ferroelect Freq Contr. 2000;47:1510-1519.

12. Wennerstrom E, Stepinski T. Model-based correction of diffraction effects of the virtual source element. IEEE Trans Ultrason Ferroelect Freq Contr. 2007;54:1614-1622.

13. Jitsumori A, Maekawa T. Mitsubishi Electric Corporation. Ultrasonic examination equipment. Japanese Patent Application S59-98288. May 16, 1984. Japanese.

14. Maekawa T, Jitsumori A, Inari T. Virtual acoustical source using a phased array. Proceedings of the SICE meeting. Jul, 1984;623-624. Japanese.

15. O'Donnell M, Thomas LJ. Efficient synthetic aperture imaging from a circular aperture with possible application to catheter-based imaging. IEEE Trans Ultrason Ferroelect Freq Contr. 1992;39: 366-380.

16. Karaman M, Li PC, O’Donnell M. Synthetic aperture imaging for small scale systems. IEEE Trans Ultrason Ferroelect Freq Contr. 1995;42: 429-442.

17. Lockwood GR, Talman JR, Brunke SS. Real-time 3-D ultrasound imaging using sparse synthetic aperture beamforming. IEEE Trans Ultrason Ferroelect Freq Contr. 1998;45:980-988.

18. Oddershede N, Jensen JA. Effects influencing focusing in synthetic aperture vector flow imaging. IEEE Trans Ultrason Ferroelect Freq Contr. 2007;54:1811-1825. 
19. Haun MA, Jones DL, O’Brien WD Jr. Efficient three-dimensional imaging from a small cylindrical aperture. IEEE Trans Ultrason Ferroelect Freq Contr. 2002;49:861-870.

20. Sumi C, Suzuki A, Nakayama K. Estimation of shear modulus distribution in soft tissue from strain distribution. IEEE Trans Biomed Eng. 1995;42:193-202.
21. Sumi C. Lateral displacement measurement using steered beams with a steering angle. Reports in Medical Imaging. 2010; In press.

\section{Publish your work in this journal}

Reports in Medical Imaging is an international, peer-reviewed, open access journal publishing original research, reports, reviews and commentaries on all areas of medical imaging. The manuscript management system is completely online and includes a very quick and fair peer-review system, which is all easy to use.

Submit your manuscript here: http://www.dovepress.com/reports-in-medical-imaging-journal

\section{Dovepress}

Visit http://www.dovepress.com/testimonials.php to read real quotes from published authors. 